\title{
Duplications of the Neuropeptide Receptor VIPR2 Confer Significant Risk for Schizophrenia
}

\author{
Vladimir Vacic ${ }^{9,11}$, Shane McCarthy ${ }^{9}$, Dheeraj Malhotra ${ }^{1,2,9}$, Fiona Murray ${ }^{7,8}$, Hsun-Hua \\ Chou $^{1,2}$, Aine Peoples ${ }^{19}$, Vladimir Makarov ${ }^{12,13}$, Seungtai Yoon ${ }^{12,13}$, Abhishek \\ Bhandari $^{1,2,9}$, Roser Corominas ${ }^{2}$, Lilia M. lakoucheva ${ }^{2}$, Olga Krastoshevsky ${ }^{18}$, Verena \\ Krause $^{18}$, Verónica Larach-Walters ${ }^{23}$, David K. Welsh ${ }^{2,5,8}$, David Craig ${ }^{21}$, John R. \\ Kelsoe $^{2,4,8}$, Elliot S. Gershon ${ }^{17}$, Suzanne M. Leal ${ }^{20}$, Marie Dell Aquila ${ }^{6,7}$, Derek W. Morris ${ }^{19}$, \\ Michael Gill ${ }^{19}$, Aiden Corvin ${ }^{19}$, Paul A. Insel ${ }^{7,8}$, Jon McClellan ${ }^{14}$, Mary-Claire King ${ }^{15,16}$, \\ Maria Karayiorgou ${ }^{10}$, Deborah L. Levy ${ }^{18}$, Lynn E. DeLisi ${ }^{22}$, and Jonathan Sebat ${ }^{1,2,3,4,9,{ }^{*}}$
}

${ }^{1}$ Beyster Center for Genomics of Psychiatric Diseases, University of California, San Diego, La Jolla, CA $1020103{ }^{2}$ Department of Psychiatry, University of California, San Diego, La Jolla, CA $1020103{ }^{3}$ Department of Cellular Molecular and Molecular Medicine University of California, San Diego, La Jolla, CA $1020103{ }^{4}$ Institute for Genomic Medicine, University of California, San Diego, La Jolla, CA $1020103{ }^{5}$ Center for Chronobiology, University of California, San Diego, La Jolla, CA $1020103{ }^{6}$ Division of Medical Genetics, University of California, San Diego, La Jolla, CA 1020103 ${ }^{7}$ Department of Medicine University of California, San Diego, La Jolla, CA $1020103{ }^{8}$ Department of Pharmacology, University of California, San Diego, La Jolla, CA 1020103; Veterans Affairs San Diego Healthcare System, San Diego, CA $92161{ }^{9}$ Stanley Center for Cognitive Genomics, Cold Spring Harbor Laboratory, Cold Spring Harbor, NY $12824{ }^{10}$ Department of Psychiatry Columbia University, New York, NY $10027{ }^{11}$ Department of Computer Science, Columbia University, New York, NY $10027{ }^{12}$ Seaver Autism Center Mount Sinai School of Medicine, New York, New York $10029{ }^{13}$ Department of Psychiatry, Mount Sinai School of Medicine, New York, New York 10029 ${ }^{14}$ Department of Psychiatry, University of Washington, Seattle WA $98195{ }^{15}$ Department of Genome Sciences University of Washington, Seattle WA $98195{ }^{16}$ Department of Medicine, University of Washington, Seattle WA 98195 17Department of Psychiatry and Behavioral Neuroscience, The University of Chicago, Chicago, IL $60637^{18}$ McLean Hospital, Belmont, MA $02478{ }^{19}$ Neuropsychiatric Genetics Research Group, Institute of Molecular Medicine and Dept. of Psychiatry, Trinity College Dublin, Ireland ${ }^{20}$ Department of Molecular and Human Genetics, Baylor College of Medicine, Houston, TX $77025{ }^{21}$ Neurogenomics Division, Translational

\footnotetext{
Users may view, print, copy, download and text and data- mine the content in such documents, for the purposes of academic research, subject always to the full Conditions of use: http://www.nature.com/authors/editorial_policies/license.html\#terms

* to whom correspondence should be addressed: Jonathan Sebat jsebat@ucsd.edu Departments of Psychiatry and Cellular \& Molecular Medicine Institute for Genomic Medicine University of California, San Diego La Jolla, CA 92093.

Author Contributions V.V and J.S. wrote the manuscript. V.V., S.M., D.M., H.H., F.M., V.M., S.Y., S.M.L., P.A.I. and J.S. designed the analytical strategy and analyzed the data. M.G., A.C., J.M., M.K., D.L.L., V.L-W., and L.E.D oversaw the recruitment and clinical assessment of study participants. M.D.A. performed cytogenetic analysis. A.B., A.P., and D.M. designed genotype assays and performed genotyping. H.H. and R.C. performed mRNA studies, F.M. performed biochemical studies. O.K., V.K., D.W.M, V.LW, L.E.D., and M.K. contributed to the interpretation of clinical patient data. J.M., M.C.K, M.K., D.L.L., L.E.D., D.C., J.R.K, and E.S.G. contributed to the interpretation of data from genetic studies. P.A.I., L.M.I., and D.K.W contributed to the interpretation of data from functional studies. J.S. coordinated the study.

Competing interests statement The authors declare no competing financial interests.
} 
Genomics Research Institute, Phoenix, Arizona $85004{ }^{22}$ Department of Psychiatry, Boston VA Healthcare System and Harvard Medical School, Brockton, MA $02301{ }^{23}$ Faculty of Medicine, Universidad Andrés Bello, Santiago, Chile

\section{Abstract}

Rare copy number variants (CNVs) play a prominent role in the etiology of schizophrenia and other neuropsychiatric disorders ${ }^{1}$. Substantial risk for schizophrenia is conferred by large $(>500$ $\mathrm{kb}$ ) CNVs at several loci, including microdeletions at $1 \mathrm{q} 21.1^{2}, 3 \mathrm{q} 29^{3}, 15 \mathrm{q} 13.3^{2}$ and $22 \mathrm{q} 11.2^{4}$ and microduplication at $16 \mathrm{p} 11.2^{5}$. However, these CNVs collectively account for a small fraction (2-4\%) of cases, and the relevant genes and neurobiological mechanisms are not well understood. Here we performed a large two-stage genome-wide scan of rare CNVs and report the significant association of copy number gains at chromosome $7 \mathrm{q} 36.3$ with schizophrenia $\left(\mathrm{P}=4.0 \times 10^{-5}, \mathrm{OR}=\right.$ $16.14[3.06, \infty])$. Microduplications with variable breakpoints occurred within a $362 \mathrm{~kb}$ region and were detected in 29 of $8,290(0.35 \%)$ patients versus two of 7,431 (0.03\%) controls in the combined sample $(\mathrm{p}$-value $=5.7 \times 10-7$, odds ratio $(\mathrm{OR})=14.1[3.5,123.9])$. All duplications overlapped or were located within $89 \mathrm{~kb}$ upstream of the vasoactive intestinal peptide receptor VIPR2. VIPR2 transcription and cyclic-AMP signaling were significantly increased in cultured lymphocytes from patients with microduplications of 7q36.3. These findings implicate altered VIP signaling in the pathogenesis of schizophrenia and suggest VIPR2 as a potential target for the development of novel antipsychotic drugs.

A majority of the rare CNVs that have been implicated in schizophrenia involve large (> 500 $\mathrm{Kb})$ genomic regions where local segmental duplication architecture promotes frequent and nearly identical rearrangements by non-allelic homologous recombination (NAHR). Because of the high structural mutation rates at these loci, the strong phenotypic effects of the causal variants, and the excellent power of most array platforms to detect such large CNVs, these genomic hotspots were the first to be detected in studies of CNVs in schizophrenia. Since most of the genome lacks the duplication architecture of the NAHR hotspots described above and because a variety of mutational mechanisms can give rise to structural rearrangements, causal variants in other regions of the genome may consist of CNVs that are individually more rare and smaller $(<500 \mathrm{~kb})$ than those arising at NAHR hotspots. For example, microdeletions of the gene Neurexin-1 (NRXN1), which are highly enriched in autism and schizophrenia ${ }^{6,7}$, consist of overlapping deletions with non-recurrent breakpoints. NRXN1 deletions are not flanked by segmental duplications, and may occur by different mutational mechanisms such as non-homologous end joining (NHEJ) or DNA replication-mediated rearrangement.

In order to identify novel schizophrenia genes, we investigated copy number variation genome-wide using an approach that detects enrichment of multiple overlapping rare variants. Regions of interest were defined in a primary sample of 802 patients and 742 controls as genomic segments containing CNVs in at least two cases and in no controls. This discovery step yielded 114 genomic regions of interest. In the secondary cohort of 7,488 patients and 6,689 controls, we assessed the association of these regions with schizophrenia 
(Supplementary Table 2). All CNVs overlapping each of the 114 regions of interest were collected, and CNV breakpoints falling within each region were used to partition the region into a series of non-overlapping segments or bins (see Supplementary Fig. 1). Significance was tested within each bin by the exact conditional test, with ethnicity and study as covariates. The segment with the minimal p-value was defined as the peak of association within the region, and a permutation-based multiple testing correction scheme was applied in order to obtain the p-value for the region.

Of the 114 regions detected in the first step, four had statistically significant associations in the secondary sample after Bonferroni correction $\left(\alpha=0.05 / 114=4.4 \times 10^{-4}\right)$. Table 1 lists the four regions with significant $\mathrm{p}$-values meeting this criterion and an additional four loci with nominally significant p-values $(\mathrm{P}<0.05)$ in the secondary cohort. Regions with significant associations were loss of copy number at 22q11.2 $\left(\mathrm{P}<5 \times 10^{-6}, \mathrm{OR}=14.2\right)$, gain at 7q36.3 $\left(\mathrm{P}=4.0 \times 10^{-5}, \mathrm{OR}=16.4\right)$, gain at $16 \mathrm{p} 11.2\left(\mathrm{P}=1.0 \times 10^{-4}, \mathrm{OR}=16.1\right)$ and loss at $15 \mathrm{q} 13.3\left(\mathrm{P}=1.6 \times 10^{-4}, \mathrm{OR}=14.9\right)$. No significant heterogeneity was observed for these genomic regions across studies (Breslow-Day-Tarone $\mathrm{P}=0.42-0.83$ ).

15q13.3, 16p11.2 and 22q11.2 are well-documented loci conferring increased risk for schizophrenia ${ }^{2,5,8}$. All are hotspots for non-allelic homologous recombination (NAHR), and all alleles contributing to the association consist of large deletions with similar breakpoints. By contrast, microduplications at $7 \mathrm{q} 36.3$ have not been previously implicated in neuropsychiatric disorders. The 7q36.3 region harbored CNVs that overlapped but differed in size and breakpoint positions (Fig. 1a). The peak of association is located in the subtelomeric region of 7q, upstream of the gene VIPR2. Also, ranking fifth among the associations genome-wide was another region, $125 \mathrm{~kb}$ proximal to the peak at $7 \mathrm{q} 36.3(\mathrm{P}=$ 0.0007 , Table 1). Combining the two $7 \mathrm{q} 36.3$ regions into a single $362 \mathrm{~kb}$ region (chr7:158,448,321-158,810,016), duplications were detected in 29 of 8,290 (0.35\%) patients and 2 of 7,431 $(0.03 \%)$ controls in this study. The p-value for the combined region in the combined sample was $5.7 \times 10^{-7}$ and the OR was 14.1 [3.5, 123.9]. A complete list of $7 \mathrm{q} 36.3$ CNVs is provided in Supplementary Table 3.

We examined sensitivity and specificity of CNV calls in the $7 q 36$ region to determine the possibility of a spurious association (Supplementary Note). No additional duplications $>100 \mathrm{~kb}$ were detected after reducing the stringency of our CNV filtering criteria. Second, identical CNV calls were obtained using a more sensitive targeted CNV calling algorithm, median Z-score Outlier Detection (MeZOD) ${ }^{5}$, (Fig. 1c-f). All but one of the duplications (control sample 06C52730) were confirmed using the Sequenom MASSarray genotyping platform with assays designed for the proximal region (Fig. 1g) and for the distal region (Fig. 1h).Validated CNVs discovered in the MGS subjects were mapped at higher resolution using the NimbleGen HD2 platform, and plots of probe intensity ratios from the HD2 array are shown in Figure 1b and Supplementary Figure 3. In addition, tandem duplications of the VIPR2 gene were confirmed in two patients by fluorescence in situ hybridization (FISH) (Supplementary Figure 4).

Unexpectedly, manual examination of probe ratios in Figure 1b revealed additional structural complexity within some of the 7q36.3 CNVs. Copy number profiles in four 
patients (03C23250, 05C43079, 03C23091 and 00C02204) suggested triplications nested within duplications of the proximal region (Fig. 1b). In all four patients, a triplication overlapped with exons 3 and 4 of the gene VIPR2. A copy number of four was confirmed in these samples using the Sequenome MASSarray CNV assay (Fig. 1g), and results for all samples were consistent with results in Figure 1b. VIPR2 transcripts were amplified from mRNA samples from the four triplication carriers. The normal VIPR2 transcript was detected in all samples, and we did not observe a larger product corresponding to a transcript with duplicated exons.

Inheritance of the duplication at 7q36.3 could be evaluated in three families (Fig. 2). In family 02-135, the duplication was confirmed in the proband, but not detected in either of the unaffected parents, and thus is apparently de novo (Fig. 2f). In family 02-016, the duplication was detected in the proband and in a mother with a diagnosis of depression (Fig. 2d). In family LW102, the duplication was detected in the proband and in an unaffected mother. The proband's mother also had a son with a diagnosis of schizophrenia (LW-102-03) from a second marriage, but DNA was not available on this individual. Clinical psychiatric reports of patients 02-016 and 02-135 are provided in the Supplementary Note.

Variable expressivity is often characteristic of pathogenic CNVs ${ }^{5}$. We evaluated the spectrum of psychiatric phenotypes associated with 7q36.3 duplications by screening for these events in individuals with bipolar disorder or autism spectrum disorder (ASD). Microarray data were available for 2,777 patients from the Bipolar Genome Study (BiGS), for 996 ASD patients from the Autism Genome Project Consortium (AGP), and from our unpublished analyses of 114 patients with ASD using the NimbleGen HD2 platform. Microduplications of $7 \mathrm{q} 36.3$ (>100 kb in size) were detected in three of $1,110(0.27 \%)$ of patients with ASD; compared with the controls described above, $\mathrm{P}=0.018$.

Microduplications at 7q36.3 were detected in two of 2,777 (0.07\%) patients with bipolar disorder; compared with the controls, $\mathrm{P}=0.23$. These results provide preliminary evidence that the clinical phenotypes associated with $7 \mathrm{q} 36.3$ duplications may include pediatric neurodevelopmental disorders such as autism, but do not include bipolar disorder. Also worthy of note, larger chromosomal abnormalities involving $7 \mathrm{q}$ have been reported in association with neurodevelopmental disorders, including deletions of 7q36-7qter ${ }^{9,10}$ and duplications of 7q35-7qter ${ }^{11}$; a $550 \mathrm{~kb}$ duplication of 7qter (of unknown clinical relevance) has been reported in a patient with neurofibromatosis 12 .

These genetic data implicate the gene VIPR2. All variants contributing to the observed association at 7q36.3 overlap with this gene or lie within the gene-less subtelomeric region $<89 \mathrm{~kb}$ from the transcriptional start site of VIPR2. VIPR2 encodes the vasoactive intestinal peptide (VIP) receptor VPAC2, a G protein-coupled receptor that is expressed in a variety of tissues including, in the brain, the suprachiasmatic nucleus, hippocampus, amygdala, and hypothalamus ${ }^{13}$. VPAC2 binds VIP ${ }^{14}$, activates cyclic-AMP signaling and PKA, regulates synaptic transmission in the hippocampus ${ }^{15,16}$, and promotes the proliferation of neural progenitor cells in the dentate gyrus ${ }^{17}$. Genetic studies in mouse have established that VIP signaling plays a role in learning and memory ${ }^{18}$. VPAC 2 also plays a role in sustaining 
normal circadian oscillations in the SCN ${ }^{19}$, and VIPR2-null ${ }^{20}$ and VIPR2overexpression ${ }^{21}$ mice exhibit abnormal rhythms of rest and activity.

Cyclic-AMP signaling has been implicated in schizophrenia ${ }^{22,23}$. We hypothesized that increases in VIPR2 transcription and VPAC2-mediated cAMP signaling would be a consequence of the microduplications at 7q36.3. We thus assessed VIPR2 mRNA and cAMP accumulation in response to VIP and a VPAC2-selective agonist (BAY 55-9837) in lymphoblastoid cell lines from eight MGS study subjects: two with subtelomeric duplications, three with duplications of VIPR2, four with partial triplications, and four controls with normal copy number of the region (see Supplementary Note). VIPR2 transcripts were present at low but measurable levels in all cell lines. VIPR2 mRNA levels were significantly increased in duplication carriers compared with controls (Fig. 3a). Likewise, cAMP responses to VIP and BAY 55-9837 were significantly greater in lymphoblastoid lines from carriers as compared to controls (Fig. 3b). In contrast, we observed no group difference in cAMP accumulation in response to a different GPCR agonist, prostaglandin E2, thus confirming that the effect of $7 \mathrm{q} 36$ duplications on cAMP accumulation is mediated by VPAC2R.

The expression patterns that we observe suggest that a variety of different genomic duplications can influence the transcription of VIPR2. The exact genetic mechanism for this is unclear. Given that some risk variants are upstream of the gene and others are complex rearrangements that could potentially disrupt the duplicate copy, our results cannot be explained simply by an increase in gene dosage. It is likely that duplications of 7q36 impact the regulation of VIPR2. Tandem duplication of regulatory sequences, for instance, could affect expression of the gene. Alternatively, the subtelomeric location of VIPR2 could be relevant to the mechanism. Intrinsic regulation of telomere structure and function often impacts the transcriptional regulation of adjacent genes, a phenomenon known as telomere position effect (TPE) ${ }^{24,25}$. If VIPR2 is under such epigenetic regulation, any large tandem duplication of the subtelomeric region could potentially cause the gene to escape repression. However, further studies are needed to determine the mechanism by which structural variants influence VIPR2 expression.

In light of the emerging roles of VIPR2 in the brain, our results support the hypothesis that the pathogenesis of schizophrenia, in some patients, involves the dysregulation of cellular processes such as adult neurogenesis and synaptic transmission and of the corresponding cognitive processes of learning and memory. Furthermore, in light of the brain expression patterns of VIPR $2^{13}$, our results support the involvement of certain brain regions, such as hippocampus, amygdala and suprachiasmatic nucleus. The link between VIPR2 duplications and schizophrenia may have significant implications for the development of molecular diagnostics and treatments for this disorder. Genetic testing for duplications of the 7q36 region could enable the early detection of a subtype of patients characterized by overexpression of VIPR2. Significant potential also exists for the development of therapeutics targeting this receptor. For instance, a selective antagonist of VPAC2R could have therapeutic potential in patients who carry duplications of the VIPR2 region. Peptide derivatives ${ }^{26}$ and small molecules ${ }^{27}$ have been identified that are selective VPAC2 inhibitors, and these pharmacological studies offer potential leads in the development of new 
drugs. While duplications of VIPR2 account for a small percentage of patients, the rapidly growing list of rare CNVs that are implicated in schizophrenia suggests that this psychiatric disorder is, in part, a constellation of multiple rare diseases ${ }^{1}$. This knowledge, along with a growing interest in the development of drugs targeting rare disorders ${ }^{28}$, provides an avenue for the development of new treatments for schizophrenia.

\section{METHODS SUMMARY}

\section{Cohort description}

Our primary cohort consisted of unrelated patients derived from family-based studies conducted by investigators at the University of Washington, McLean Hospital, Columbia University, Trinity College Dublin, NYU and Harvard Medical Schools (Supplementary Table 1). All samples were analyzed by array CGH using the NimbleGen HD2 platform. The secondary cohort consisted of Affymetrix SNP Array 6.0 data from the MGS study of schizophrenia ${ }^{29}$, publicly available data from the International Schizophrenia Consortium (ISC), genotyped using Affymetrix 6.0 and 5.0 platforms ${ }^{2}$, and Affymetrix 6.0 data on an independent set of controls from the Bipolar Genome Study (BiGS) ${ }^{30}$ (Supplementary

Table 1).

\section{Intensity data processing and rare CNV calling}

With the exception of the published CNV calls from the ISC, all data were processed and analyzed centrally as follows. Microarray intensity data were normalized, and CNV calls were generated using an analysis package that we developed called C-score. All CNV call sets were filtered in a consistent fashion. In order to minimize the differential sensitivity of the various array platforms to detect CNVs, we limited our analysis to CNVs $>100 \mathrm{~kb}$. This size range is readily detectable by all platforms used in this study. The same criteria have been previously applied to filter CNVs across studies ${ }^{2}$. Last, sensitivity to detect large $(>100 \mathrm{~kb})$ copy number polymorphisms (CNPs) was evaluated at several locations in the genome. Overall sensitivity to detect CNVs was comparable in cases and controls in both cohorts. Additional details regarding $\mathrm{C}$-score, statistical methods and evaluation of $\mathrm{CNV}$ call sets are described in the Supplementary Note.

Methods and any associated references are available in the online version of the paper and in the Supplementary Note.

\section{Supplementary Material}

Refer to Web version on PubMed Central for supplementary material.

\section{ACKNOWLEDGMENTS}

This study was supported by a gift from Ted and Vada Stanley to the Cold Spring Harbor Laboratory, a gift to J.S. from the Beyster family foundation, NIH grants to J.S. (MH076431, HG04222), D.L.L. (MH071523), M.C.K (MH083989), P.I. (GM66232), F.M. (HL091061), D.K.W. (MH082945), M.K. (MH061399), L.E.D (MH044245), grants to J.S., D.K.W., D.L.L. and M.C.K from NARSAD,.grants to A.C., M.G., D.M. from the Wellcome Trust (072894/Z/03/Z) and Science Foundation Ireland (08INIB1916), a career development award to D.K.W. by the Veterans Administration, and grants to D.L.L. from the Sidney R,. Baer, Jr. Foundation and Essel Foundation . We wish to thank the Genetic Association Information Network (GAIN), Molecular Genetics of Schizophrenia (MGS) 
and the Bipolar Genome Study (BiGS) for providing data for this study (investigators listed in the Supplementary Note). We wish to thank Barbara Trask, Robert Malinow, and Joseph Gleeson for helpful discussions.

\section{References}

1. Sebat J, Levy DL, McCarthy SE. Rare structural variants in schizophrenia: one disorder, multiple mutations; one mutation, multiple disorders. Trends Genet. 2009; 25:528-535. [PubMed: 19883952]

2. International Schizophrenia Consortium. Rare chromosomal deletions and duplications increase risk of schizophrenia. Nature. 2008; 455:237-241. [PubMed: 18668038]

3. Mulle JG, et al. Microdeletions of 3q29 confer high risk for schizophrenia. Am J Hum Genet. 2010; 87:229-236. [PubMed: 20691406]

4. Karayiorgou M, et al. Schizophrenia susceptibility associated with interstitial deletions of chromosome 22q11. Proc Natl Acad Sci U S A. 1995; 92:7612-7616. [PubMed: 7644464]

5. McCarthy SE, et al. Microduplications of 16p11.2 are associated with schizophrenia. Nat Genet. 2009; 41:1223-1227. [PubMed: 19855392]

6. Szatmari P, et al. Mapping autism risk loci using genetic linkage and chromosomal rearrangements. Nat Genet. 2007; 39:319-328. [PubMed: 17322880]

7. Rujescu D, et al. Disruption of the neurexin 1 gene is associated with schizophrenia. Hum Mol Genet. 2008

8. Stefansson H, et al. Large recurrent microdeletions associated with schizophrenia. Nature. 2008; 455:232-236. [PubMed: 18668039]

9. Tyson C, et al. Submicroscopic deletions and duplications in individuals with intellectual disability detected by array-CGH. Am J Med Genet A. 2005; 139:173-185. [PubMed: 16283669]

10. Wu Y, et al. Submicroscopic subtelomeric aberrations in Chinese patients with unexplained developmental delay/mental retardation. BMC Med Genet. 2010; 11:72. [PubMed: 20459802]

11. Morava E, et al. Small inherited terminal duplication of $7 \mathrm{q}$ with hydrocephalus, cleft palate, joint contractures, and severe hypotonia. Clin Dysmorphol. 2003; 12:123-127. [PubMed: 12868476]

12. Bartsch $\mathrm{O}$, et al. Two independent chromosomal rearrangements, a very small $(550 \mathrm{~kb})$ duplication of the $7 \mathrm{q}$ subtelomeric region and an atypical 17q11.2 (NF1) microdeletion, in a girl with neurofibromatosis. Cytogenet Genome Res. 2007; 119:158-164. [PubMed: 18160797]

13. Sheward WJ, Lutz EM, Harmar AJ. The distribution of vasoactive intestinal peptide 2 receptor messenger RNA in the rat brain and pituitary gland as assessed by in situ hybridization. Neuroscience. 1995; 67:409-418. [PubMed: 7675176]

14. Fahrenkrug J. Transmitter role of vasoactive intestinal peptide. Pharmacol Toxicol. 1993; 72:354363. [PubMed: 8103215]

15. Yang K, et al. Vasoactive intestinal peptide acts via multiple signal pathways to regulate hippocampal NMDA receptors and synaptic transmission. Hippocampus. 2009; 19:779-789. [PubMed: 19173226]

16. Waschek JA. Vasoactive intestinal peptide: an important trophic factor and developmental regulator? Dev Neurosci. 1995; 17:1-7. [PubMed: 7621745]

17. Zaben M, et al. The neurotransmitter VIP expands the pool of symmetrically dividing postnatal dentate gyrus precursors via VPAC2 receptors or directs them toward a neuronal fate via VPAC1 receptors. Stem Cells. 2009; 27:2539-2551. [PubMed: 19650041]

18. Chaudhury D, Loh DH, Dragich JM, Hagopian A, Colwell CS. Select cognitive deficits in vasoactive intestinal peptide deficient mice. BMC Neurosci. 2008; 9:63. [PubMed: 18616823]

19. Brown TM, Colwell CS, Waschek JA, Piggins HD. Disrupted neuronal activity rhythms in the suprachiasmatic nuclei of vasoactive intestinal polypeptide-deficient mice. J Neurophysiol. 2007; 97:2553-2558. [PubMed: 17151217]

20. Harmar AJ, et al. The VPAC(2) receptor is essential for circadian function in the mouse suprachiasmatic nuclei. Cell. 2002; 109:497-508. [PubMed: 12086606]

21. Underhill PA, et al. Y chromosome sequence variation and the history of human populations. Nat Genet. 2000; 26:358-361. [PubMed: 11062480] 
22. Millar JK, et al. DISC1 and PDE4B are interacting genetic factors in schizophrenia that regulate cAMP signaling. Science. 2005; 310:1187-1191. [PubMed: 16293762]

23. Turetsky BI, Moberg PJ. An odor-specific threshold deficit implicates abnormal intracellular cyclic AMP signaling in schizophrenia. Am J Psychiatry. 2009; 166:226-233. [PubMed: 19074977]

24. Gottschling DE, Aparicio OM, Billington BL, Zakian VA. Position effect at S. cerevisiae telomeres: reversible repression of Pol II transcription. Cell. 1990; 63:751-762. [PubMed: 2225075]

25. Koering CE, et al. Human telomeric position effect is determined by chromosomal context and telomeric chromatin integrity. EMBO Rep. 2002; 3:1055-1061. [PubMed: 12393752]

26. Moreno D, et al. Development of selective agonists and antagonists for the human vasoactive intestinal polypeptide VPAC(2) receptor. Peptides. 2000; 21:1543-1549. [PubMed: 11068102]

27. Chu A, Caldwell JS, Chen YA. Identification and characterization of a small molecule antagonist of human VPAC(2) receptor. Mol Pharmacol. 2009; 77:95-101. [PubMed: 19854890]

28. Braun MM, Farag-El-Massah S, Xu K, Cote TR. Emergence of orphan drugs in the United States: a quantitative assessment of the first 25 years. Nat Rev Drug Discov. 2010; 9:519-522. [PubMed: 20531273]

29. Shi J, et al. Common variants on chromosome $6 \mathrm{p} 22.1$ are associated with schizophrenia. Nature. 2009; 460:753-757. [PubMed: 19571809]

30. Zhang D, et al. Singleton deletions throughout the genome increase risk of bipolar disorder. Mol Psychiatry. 2008 
A

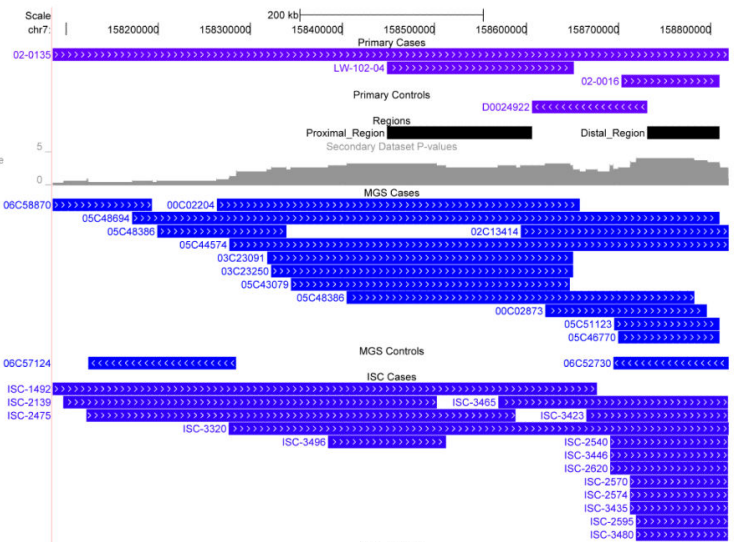

C
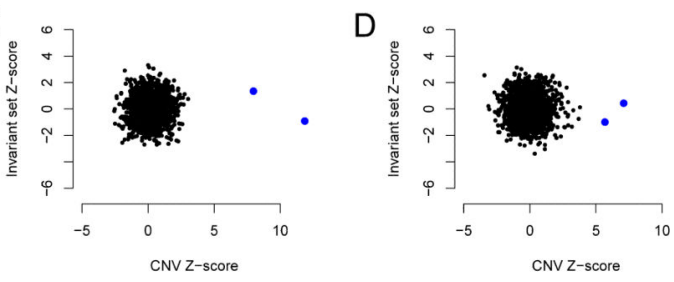

E

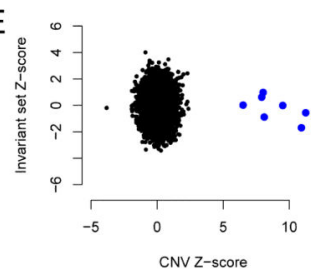

F

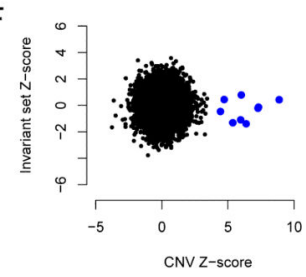

G

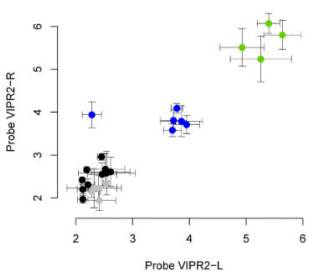

$\mathrm{H}$

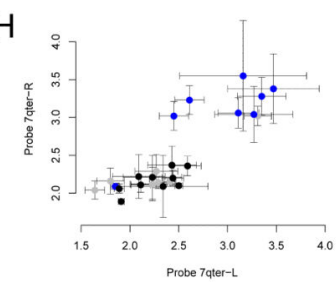

LW-102-04

02-0016
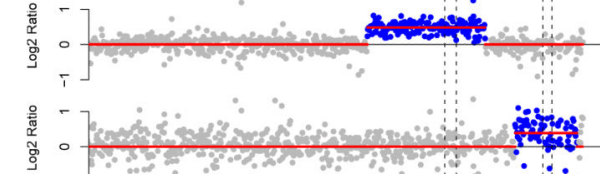

* D0024922

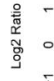
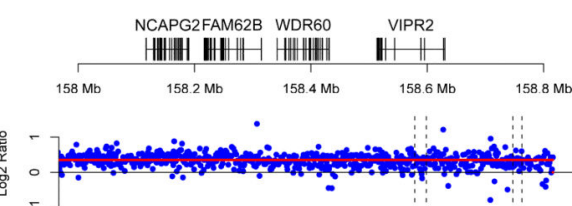

5C48694

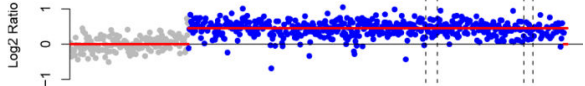

05C48386

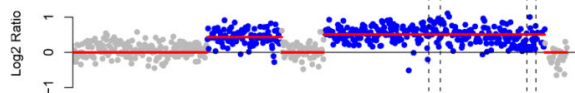

$05 C 44574$

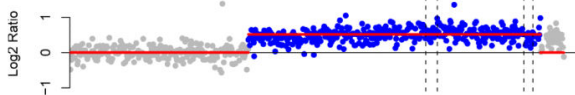

$03 \mathrm{C} 23250$

势

05C43079

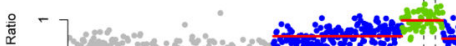

0

03С23091

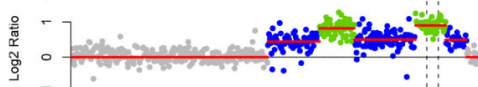

00602204

竞

$02 C 13414$

然

00602873

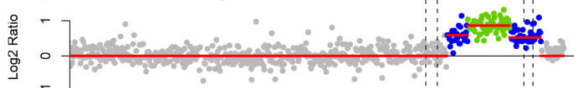

05C51123

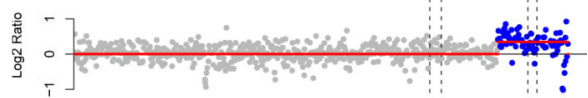

05C46770

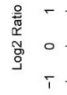

* $06 \mathrm{C} 52730$

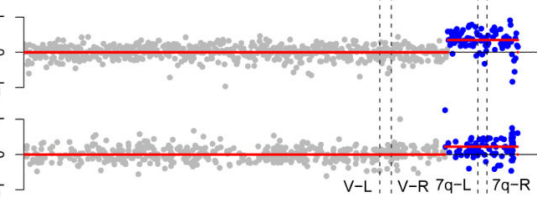

然

Figure 1. Detection and validation of microduplications and triplications of $7 q 36.3$

a) Map of CNVs detected in the primary and secondary cohorts from the UCSC genome browser. (b) Plots of probe intensity ratios for $16 \mathrm{CNVs}$ detected in the primary and MGS datasets. All are cases, with the exception of two controls, who are indicated with an asterisk*. Regions with estimated copy numbers of 2, 3 and 4 are highlighted in gray, blue and green, respectively. Locations of four Sequenom validation assays are shown (dashed lines). (c-f) CNV genotypes were confirmed by MeZOD cluster plots of probe intensity ratios of the proximal and distal regions and in the primary dataset (c and d, respectively) 
and secondary dataset (e and f, respectively). Absolute copy numbers were confirmed for duplications and triplications of the proximal $(\mathrm{g})$ and distal $(\mathrm{h})$ regions by Sequenom MASSarray genotyping. 
a

LW102

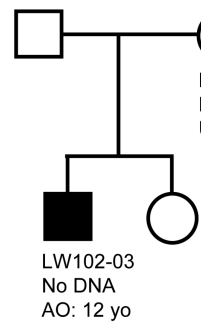

d

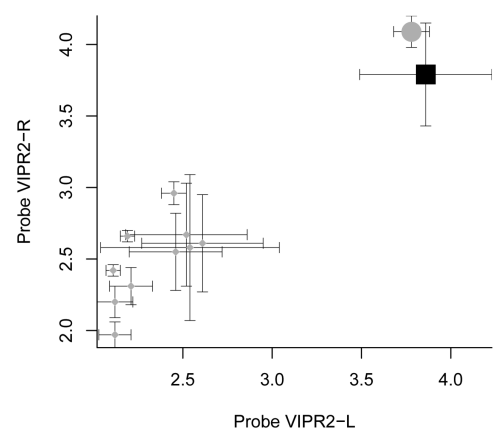

b

LW102-04

Dup 12 yo

e

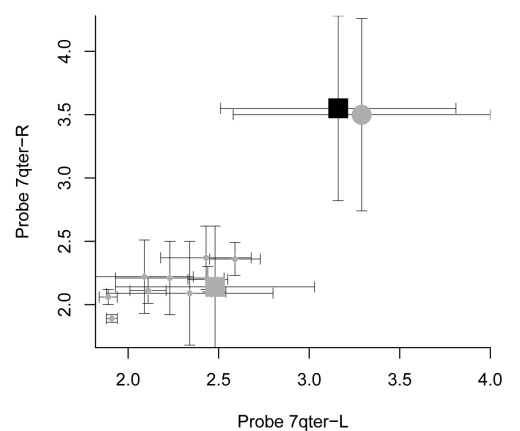

MK016

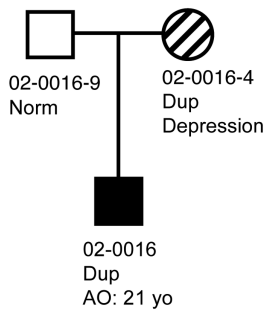

C

MK135

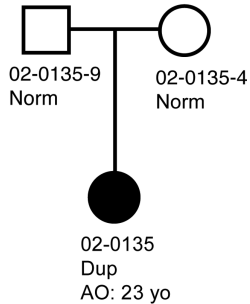

f

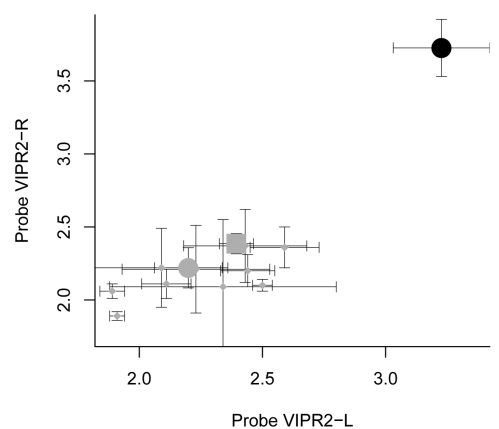

Figure 2. Patterns of CNV inheritance in families

Pedigree diagrams are shown for families (a) LW102, (b) 02-016 and (c) 02-135, along with the Sequenom validation for families (d) LW102, (e) 02-016 and (f) 02-135. Sequenom validation was performed on (a) mother and one of the affected sons, and (b) all three family members, along with $10 \mathrm{CEU}$ HapMap controls. Sequenom assays confirmed that duplications were present in the patients and maternally inherited from LW102-2 and 02-0016-4. 

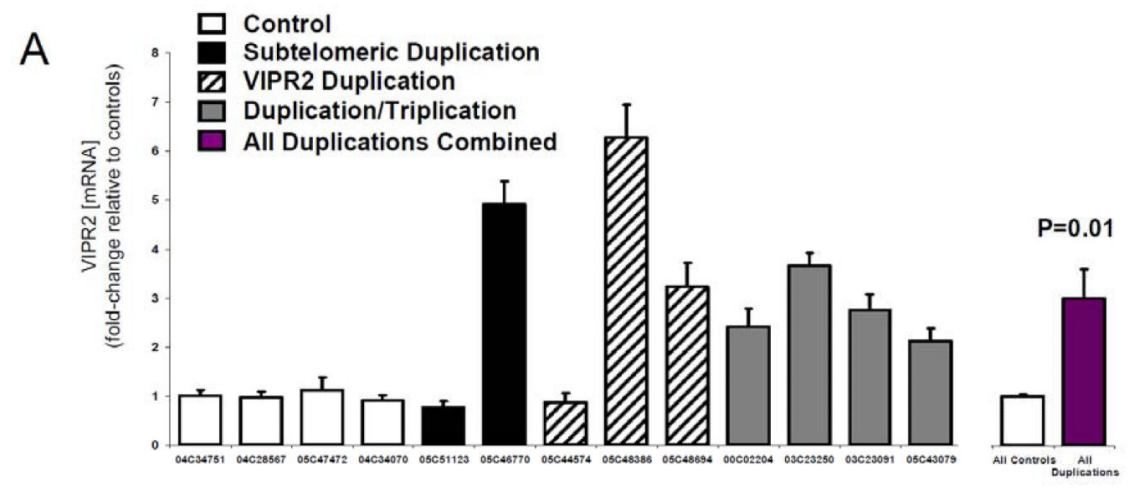

B

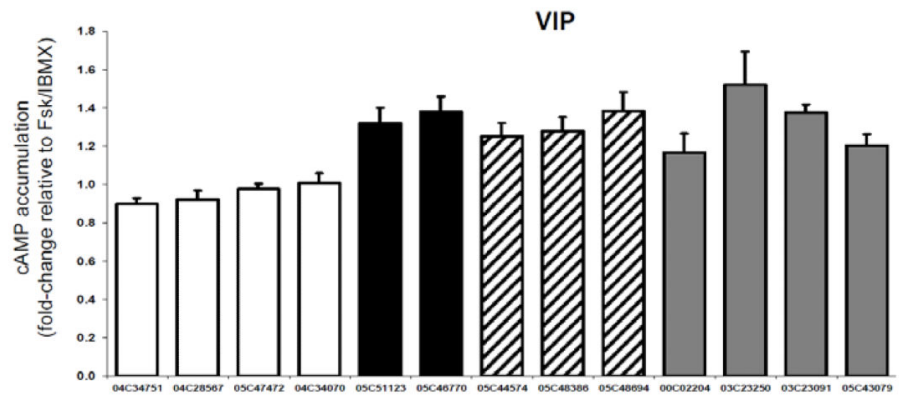

$\mathrm{P}<0.0001$

C
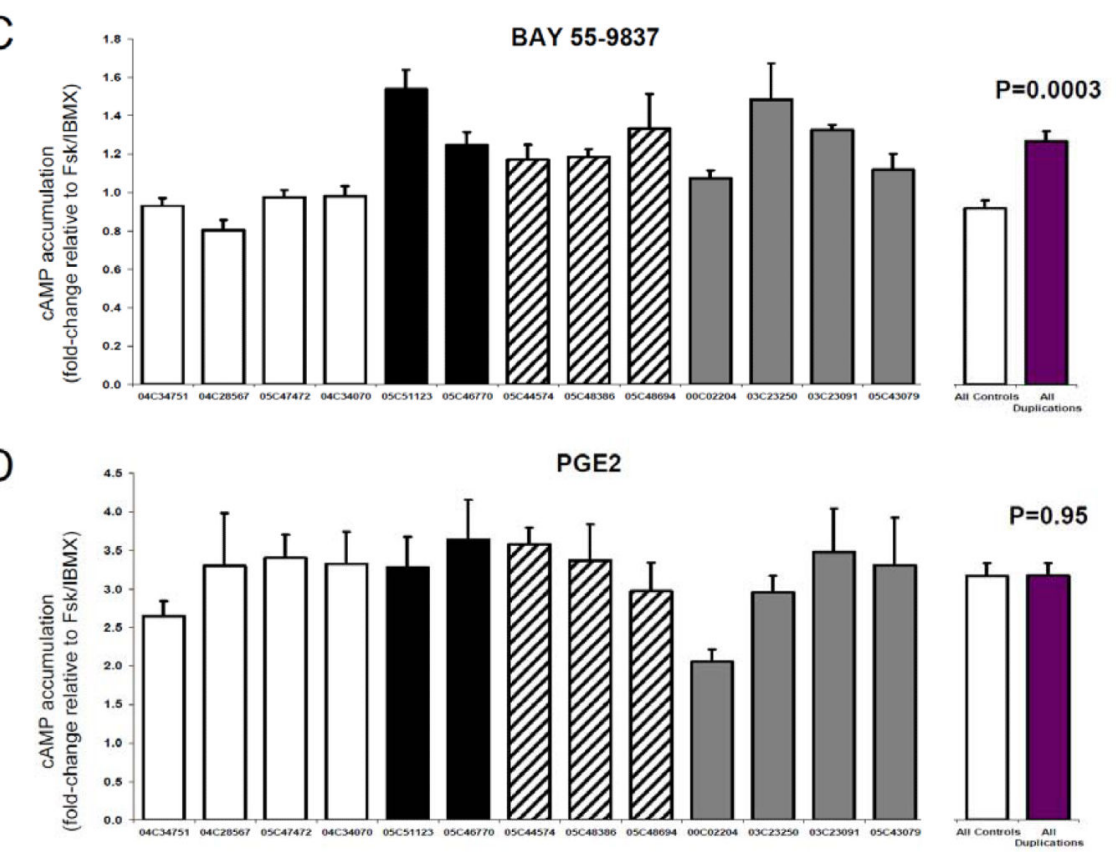

Figure 3. Duplications and triplications of 7q36.3 result in increased VIPR2 transcription and cyclic-AMP signaling

(a) Quantitative PCR results of VIPR2 mRNA from lymphoblastoid cell lines. Two to four subjects were tested for each of four genotypes (subtelomeric duplication, VIPR2

duplication, exon 3/4 triplication, and normal diploid copy number as control). Results are expressed as the mean fold-change of CNV carriers relative to the mean of control samples. (b-c) Cyclic AMP accumulation was measured in the same cell lines in response to VIP (100nM) and the VPAC2 agonist BAY 55-9837 (100nM). Results are expressed as foldchange over forskolin/IBMX alone. (d) No significant differences were observed in cAMP 
response to another GPCR agonist, Prostaglandin E2 (PGE2, $1 \mu \mathrm{M}$ ), demonstrating that the effects are specific to VPAC2. For subjects, error bars represent standard error of the mean computed across replicates. Differences between the groups of 9 duplication carriers and 4 controls were tested using unpaired two sample t-tests. 


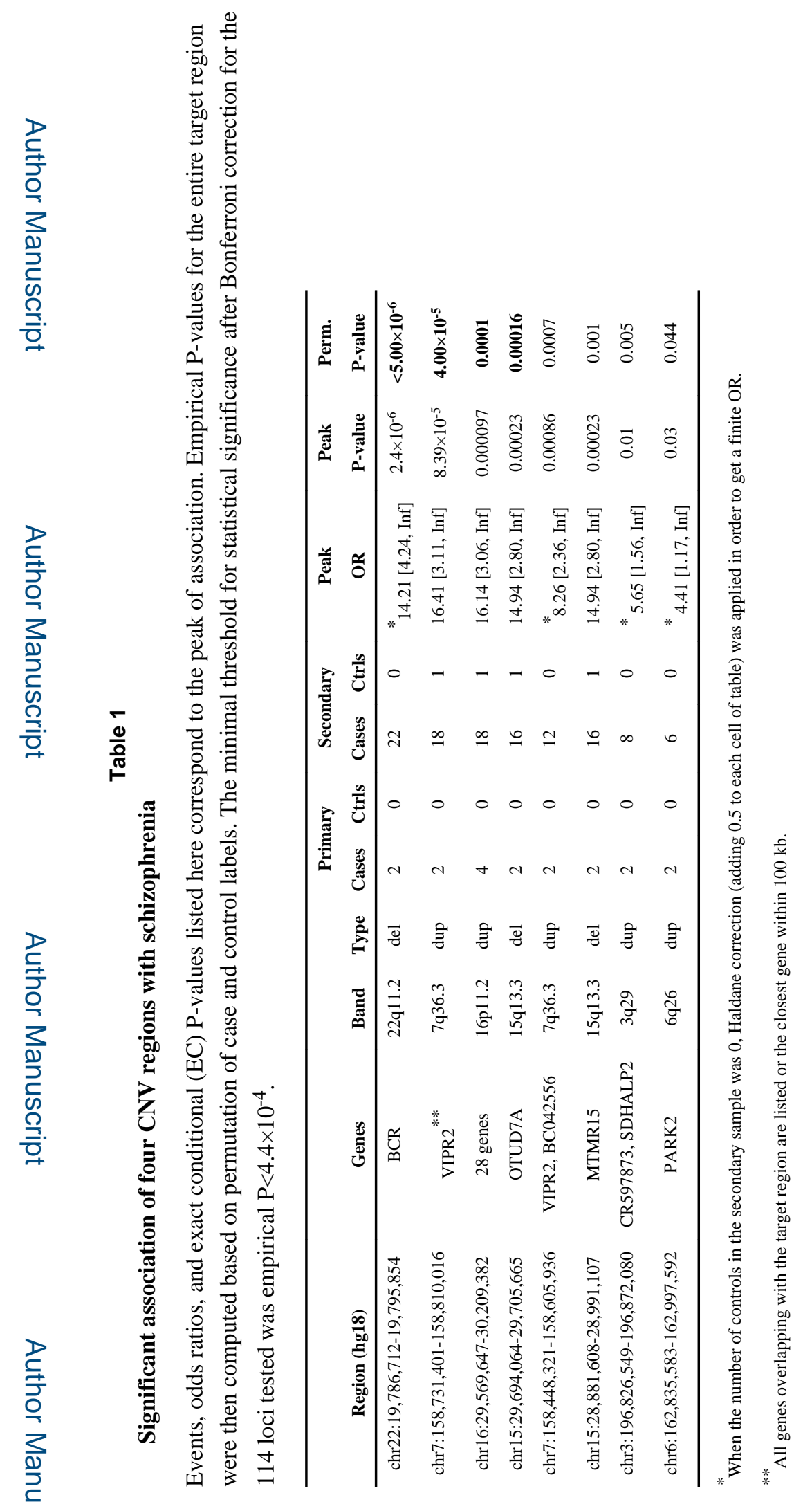

Nature. Author manuscript; available in PMC 2012 May 14. 